
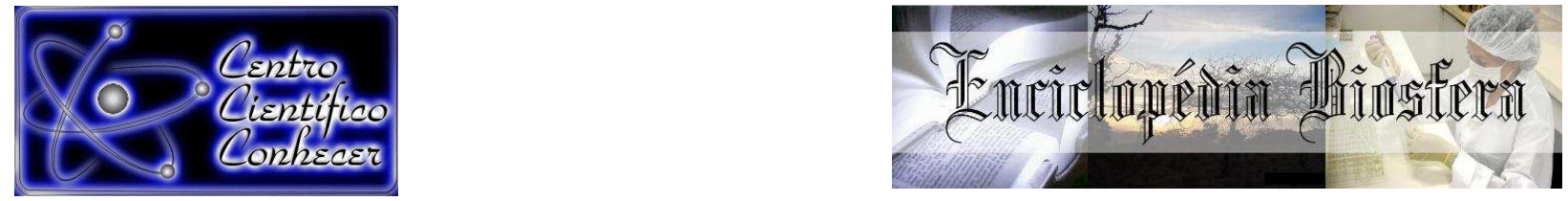

\title{
NIVELAMENTO DO PLATÔ TIBIAL BASEADO NO CORA E AVANÇO DA TUBEROSIDADE DA TÍBIA PARA TRATAMENTO DE LESÃO DO LIGAMENTO CRUZADO CRANIAL- RELATO DE CASO
}

George Willanne Mota dos Santos Mercês'; Francisco Assis Dorea Neto; João Victo Romano Vieria ${ }^{3}$, João Moreira Costa Neto ${ }^{2}$, Arianne Pontes Oriá ${ }^{2}$

1. Residente do Programa de Residência em Cirurgia de Carnívoros Domésticos, Departamento de Anatomia, Patologia, Escola de Medicina Veterinária e Zootecnia, Universidade Federal da Bahia Salvador - Brasil.

2 .Professor Doutor do Departamento de Anatomia, Patologia, Escola de Medicina Veterinária e Zootecnia, Universidade Federal da Bahia (Francisco.dorea@ufba.br) 3 .Mestrando do Programa de pós-graduação Ciência Animal nos Trópicos. Escola de Medicina Veterinária e Zootecnia, Universidade Federal da Bahia.

Recebido em: 08/09/2015 - Aprovado em: 14/11/2015 - Publicado em: 01/12/2015 DOI: http://dx.doi.org/10.18677/Enciclopedia_Biosfera_2015_253

\section{RESUMO}

Neste estudo relata-se a utilização da osteotomia de nivelamento baseado no CORA (osteotomia baseada no centro de rotação angular) CBLO unido ao princípio da cirurgia de osteotomia tripla da tíbia (TTO), para obtenção da rotação cranial da tíbia e o avanço da tuberosidade da tibial no tratamento da deficiência do ligamento cruzado cranial em um canino de $24 \mathrm{Kg}$. A mensuração do ângulo do platô tibial (TPA) foi realizado através de projeções radiográficas. O ângulo de correção encontrado de $25^{\circ}$ resultou em um ângulo do TPA pós-operatório de $13,6^{\circ}$. Avaliações clínicas foram realizadas aos 10,20, 30 e 60 dias de pós-operatório e as avaliações radiográficas imediatamente após o ato operatório e aos 30 e 60 dias. A técnica resultante da combinação de CBLO e TTO demonstrou ser tão eficaz comparativamente às técnicas anteriores no que tange ao tratamento da deficiência do ligamento cruzado cranial por proporcionar neutralização do impulso cranial da tíbia e manutenção do ângulo do platô tibial.

PALAVRAS-CHAVE: Ângulo do platô tibial, CBLO, TTO

\section{CORA BASED LEVELING OSTEOTOMY AND TIBIAL TUBEROSITY ADVANCED FOR TREATMENT OF CRANIAL CRUCIATE LIGAMENT INJURY - CASE REPORT.}

\begin{abstract}
In this study we report the use of leveling osteotomy based on CORA (osteotomy based on the center of rotation and angulation) - CBLO combined to the principle of triple tibial osteotomy surgery (TTO) to obtain the cranial tibial rotation and
\end{abstract}


advancement of the tibial tuberosity in the treatment of cranial cruciate ligament deficiency in a dog of $24 \mathrm{~kg}$. The measurement of the tibial plateau angle (TPA) was performed by radiographic projections. The $25^{\circ}$ corre ction angle found resulted in postoperative TPA angle of $13.6^{\circ}$. Clinical assessme nts were performed at $10,20,30$ and 60 days and the radiographic examinations were conducted immediately after surgery and at 30 and 60 days. The resulting technique combining CBLO and TTO was as effective compared to the prior surgical procedures described before for the cranial cruciate ligament deficiency treatment providing neutralization of the cranial tibial thrust and maintaining the angle of the tibial plateau.

KEYWORDS: CBLO, TTO, Tibial plateau angle

\section{INTRODUÇÃO}

A deficiência do ligamento cruzado cranial DLCC é uma das lesões mais comuns no cão, sendo também a causa mais comum de claudicação em membro pélvico de cães (PIERMATTEI et al., 2006, FERRIGNO et al., 2009). É considerada a principal causa de Doença Articular Degenerativa (DAD) do joelho em caninos (PIERMATTEl et al., 2006).

O diagnóstico dessa enfermidade é balizado principalmente no histórico e no exame ortopédico, principalmente na pesquisa do movimento de gaveta e no teste de compressão tibial. A obtenção de imagens radiográficas durante a execução deste teste podem também ser de grande ajuda no diagnóstico (VASSEUR, 2007). Com a instabilidade pós ruptura, o joelho afetado começa a sofrer uma série de alterações inflamatórias, causando uma cascata de eventos que incluem o aparecimento de osteoartrite progressiva e o acometimento do menisco medial, o que leva ao aparecimento de sinais clínicos como claudicação, dor e disfunção do membro (SCHULZ, 2007, FERRIGNO et al., 2009, COOK, 2010).

Ao longo dos anos foram desenvolvidas técnicas cirúrgicas com intuito de tratar a ruptura do ligamento cruzado cranial. Essas técnicas estão divididas em: intra-articulares, técnicas extra-articulares e técnicas periaticulares (COMERFORD et al., 2013).

A osteotomia para nivelamento do platô tibial (TPLO), desenvolvida por SLOCUN em 1984, tem como objetivo ajustar o platô da tíbia para um ângulo entre $5-7^{\circ}$, limitando assim a força de cisalhamento gerad a pela compressão do joelho durante o suporte do peso, o que, por sua vez, diminui a pressão cranial da tíbia (SCHULZ, 2007, MILOVANCEV \& SCHAEFER, 2010). A técnica descrita em 2007, determinada como osteotomia tripla da tíbia (TTO), altera a conformação da tíbia proximal fazendo com que o tendão patelar fique orientado perpendicularmente ao platô tibial quando o joelho está em ângulo de suporte de peso (BRUCE et al., 2007; KIM et al., 2008, MOLES et al., 2009). RASKE et al., (2013), apresentaram uma nova proposta de osteotomia para o nivelamento do platô tíbia baseando-se no método de osteotomia determinado pelo centro de rotação angular (CORA) ou o CBLO (nivelamento baseado no CORA) como ficou conhecido. Esta técnica reduz o impulso tibial cranial em joelhos com DLCC. O centro de rotação angular dos caninos encontra-se na curvatura proximal da tíbia e, através da projeção radiográfica médio-lateral pode definido o ângulo desejado do platô tibial (RASKE et al., 2013). 
O objetivo deste relato foi descrever a utilização do método CBLO com uma variação seguindo o princípio da cirurgia de TTO, proporcionando além da rotação cranial da tíbia pelo CORA o avanço da tuberosidade da tíbia.

\section{MATERIAL E METODOS}

\section{Descrição do caso clínico}

Foi atendido no Hospital Veterinário da UFBA um canino, sem raça definida, macho, castrado de 11 anos, pesando $24 \mathrm{~kg}$, que apresentava claudicação do membro posterior esquerdo (MPE) há aproximadamente três meses. O exame físico geral não demonstrou nenhuma alteração. Ao exame ortopédico observou-se dor à palpação, teste de gaveta e de compressão tibial positivos no MPE. Ao exame radiográfico na projeção médio-lateral foi observado deslocamento cranial do platô tibial em relação aos côndilos femorais, sugestivo de deficiência do ligamento cruzado cranial e na projeção crânio-caudal foi visibilizado osteoartrose.

\section{Avaliação radiográfica e planejamento cirúrgico}

Para o planejamento cirúrgico foi consignado radiografia da articulação do joelho esquerdo em incidência médio-lateral com o membro totalmente estendido e côndilos femorais sobrepostos. O ângulo do platô tibial (TPA) foi mensurado através de projeção radiográfica conforme método relatado por REIF \& PROBST (2003) e REIF et al. (2004). O ângulo do CORA foi mensurado semelhante ao descrito por RASKE et al. (2013), pela intersecção entre os eixos da tíbia proximal e distal.

$\mathrm{O}$ ângulo de correção encontrado pós mensurações foi de $25^{\circ}$. Com isso, o ângulo de rotação foi calculado como sendo dois terços do ângulo de correção ( $A^{\circ}$ de correção $-5 \% 3 \times 2$ ). Considerando o cálculo do ângulo de rotação o resultado em milímetros da rotação segue conforme a tabela do CORA (tamanho da lâmina/ângulo de rotação). O que difere é o avanço da tuberosidade da tíbia saindo de um ângulo de $25^{\circ}$. O que resulta na obtenção de u m terço do ângulo do avanço da tuberosidade. O resultado da TPA, baseado pelo CORA no pós-operatório foi de 13,6 (FIGURA 1). 


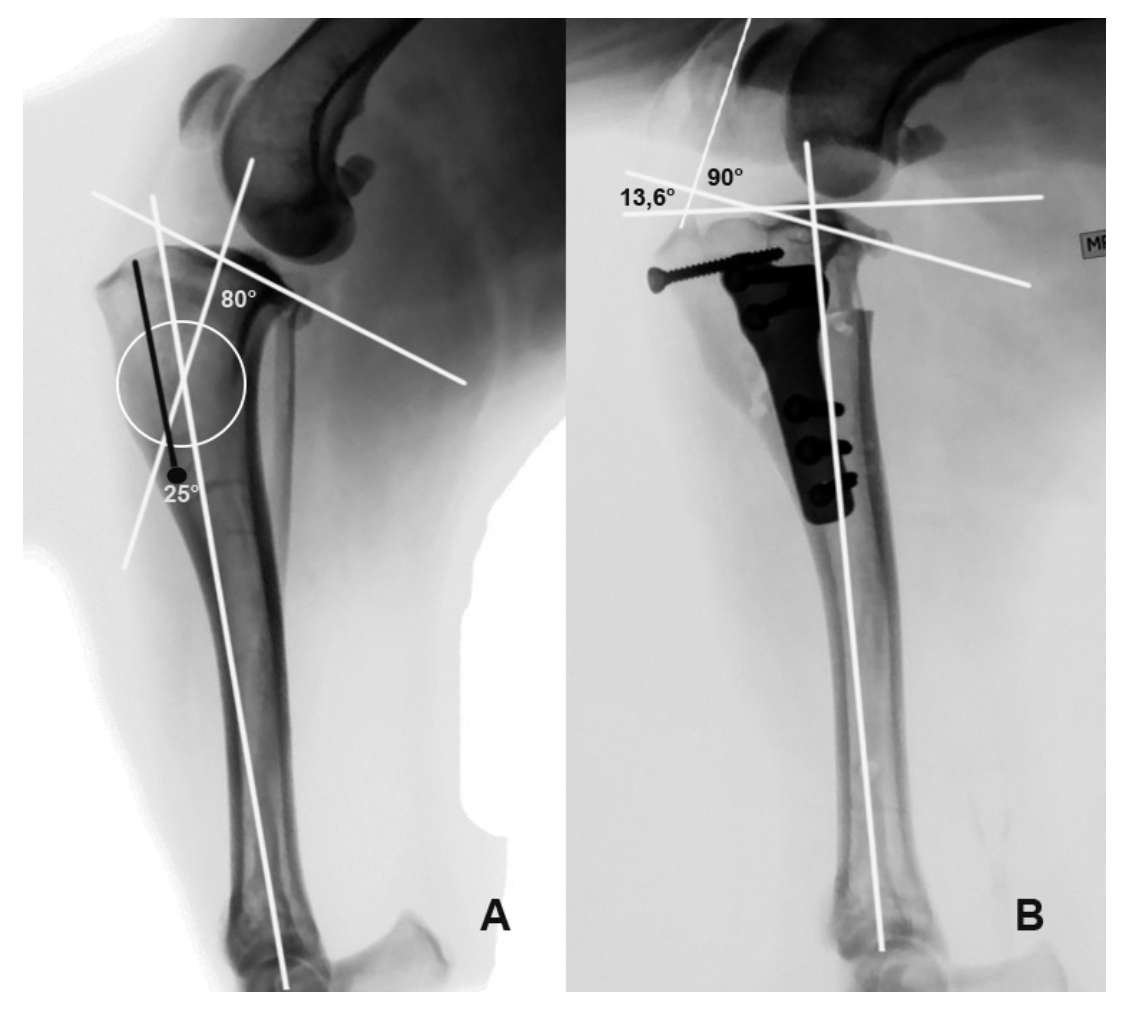

FIGURA 1. A: Planejamento radiográfico pré-operatório definindo o ângulo de correção baseado no CORA e local para osteotomia da tuberosidade da tíbia. B: Resultado do ângulo do TPA de $13,6^{\circ}$ pós Avanço da tuberosidade da tíbia e rotação do segmento.

\section{Procedimento anestésico e cirúrgico}

O paciente foi pré-medicado por via endovenosa com cloridrato de clorpromazina $(0,3 \mathrm{mg} / \mathrm{Kg})$ (Longactil®, Cristália, São Paulo, Brasil e morfina $(0,5$ $\mathrm{mg} / \mathrm{Kg}$ ) (Dimorf®, Cristalia, São Paulo, Brasil) por via intramuscular. Realizou-se tricotomia de todo o membro pélvico esquerdo e da região lombossacra, seguiu-se a indução anestésica por via endovenosa com propofol $(4 \mathrm{mg} / \mathrm{Kg})\left(\operatorname{Propovan}{ }^{\circledR}\right.$, Cristália, São Paulo, Brasil). Procedeu-se a intubação endotraqueal e a manutenção anestésica com isoflurano (Vetflurano $\AA$, Virbac, São Paulo, Brasil), diluído em oxigênio $100 \%$ em sistema semifechado. Para o bloqueio peridural foi calculado o volume final $\left(0,25 \mathrm{ml} / \mathrm{Kg}\right.$ ), sendo este composto de $3 \mathrm{ml}$ de cloridrato de lidocaína $2 \%$ (Xylestesin ${ }^{\circledR}$, Syntec, São Paulo, Brasil) sem vaso constritor, $2,8 \mathrm{ml}$ de Cloridrato de bupivacaína $0,5 \%$ (Bupican® ${ }^{\circ}$, Claris, São Paulo, Brasil) e $0,2 \mathrm{ml}$ de morfina $1 \%$. Adicionalmente utilizou-se, por via endovenosa, cefalotina $(30 \mathrm{mg} / \mathrm{Kg}$ ) (Cefalotina Sodica®), Eli Lilly, Pernambuco, Brasil)

O animal foi posicionado em decúbito dorsal levemente rotacionado para o lado do membro afetado. $O$ membro foi preparado assepticamente para a cirurgia. Foi realizada abordagem medial à articulação do joelho de acordo com a técnica descrita por PIERMATTEl et al., (2006). Ato contínuo realizou-se a curetagem do ligamento rompido e meniscectomia medial. 
A capsula articular foi suturada com fio de nylon 2.0 (Nylon, Technofio) com pontos em wolf. Prolongou-se a incisão para a face medial da tíbia, expondo a tuberosidade. O tamanho da tuberosidade da tíbia (TT) foi mensurado e em seguida realizada uma perfuração transversal de $2 \mathrm{~mm}$ caudal esta. Através deste furo foi inserido um guia de precisão para realização da osteotomia da TT com auxilio de serra oscilatória elétrica e, posteriormente, a mesma foi afastada com auxilio uma alavanca (Figura 2A). Um "JIG" improvisado como fixador externo aplicado inicialmente através de um pino de schanz foi inserido na face medial da tíbia proximal, caudal ao ligamento colateral medial, 3 a $4 \mathrm{~mm}$ distal à superfície articular. $\mathrm{O}$ pino foi alinhado perpendicularmente ao tendão patelar e paralelo à superfície articular da tíbia, para que o corpo do "JIG" permanecesse paralelo ao plano sagital da tíbia. Um segundo pino foi inserido na tíbia distal e os parafusos do fixador foram, então, apertados para a manutenção da rigidez do aparelho.

A serra oscilatória com uma lamina semi-circular $n^{0} 15$ foi posicionada caudal à crista da tíbia osteotomizada até a região do córtex caudal da tíbia, perpendicular ao plano do CORA iniciando-se a osteotomia. $O$ procedimento foi pausado na metade, primeira cortical, para que fossem realizadas as marcações de acordo às mensurações previas para atingir a rotação desejada (Figura 2B). Um pino de $3 \mathrm{~mm}$ foi inserido no segmento ósseo do platô tibial para auxiliar como ferramenta na rotação cranial deste fragmento, a parte óssea seccionada foi rotacionada cranialmente causando, assim, o avanço cranial do platô da tíbia (1/3) e do avanço da tuberosidade da tíbia (2/3) e da rotação do CORA conforme métodos empregados por BRUCE et al., (2007) e RASKE et al., (2013) e também em consoante com 0 ângulo medido anteriormente. A osteotomia foi finalizada e a rotação desejada alcançada, sendo mantida com um pino para estabilização (Figura 2C). Ato contínuo foi aplicado uma placa de TPLO (Laboratório de ortopedia veterinária - UFBA) de 2,7mm na face medial da tíbia fixando os fragmentos distal e proximal após a osteotomia. Para fixação da placa foram utilizados seis parafusos de $2.7 \mathrm{~mm}$ que se distribuíram três na porção óssea distal e os outros três na porção óssea proximal e um parafuso de $2,7 \mathrm{~mm}$ foi transfixado na TT fixando-o a tíbia (Figura 2D). 


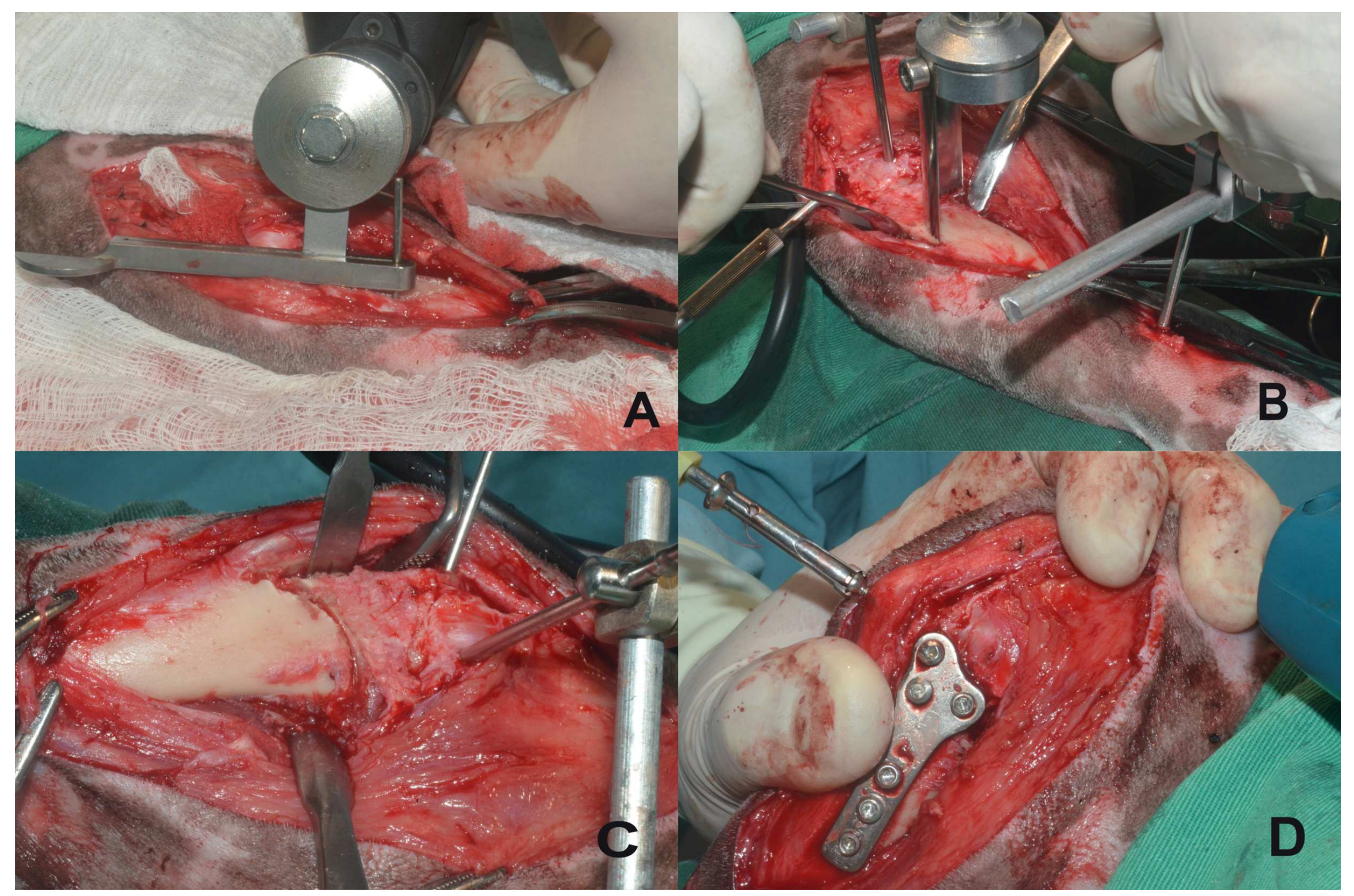

FIGURA 2. Imagens fotográficas de procedimento cirúrgico para nivelamento do platô baseado no CORA e osteotomia da tuberosidade da tíbia. A: Osteotomia da TT; B: Osteotomia da tíbia com serra semi-circular; C: Demonstração da tíbia após a osteotima da TT e da tíbia proximal; D: Fixação da tíbia com placa de TPLO e da TT com parafuso após rotação do segmento e avanço da TT.

O periósteo foi suturado com padrão contínuo simples com mononylon 2-0 seguindo da sutura do subcutâneo com padrão contínuo simples. A pele foi suturada com mononylon 3-0 com padrão em Sultan.

\section{Condutas no pós-operatório}

Como medicações pós-operatórias foram prescritos cloridrato de ranitidina (Ranitidina Xarope $\AA$, EMS, São Paulo, Brasil) (2mg/kg/BID/10dias), cefalexina (PetSporin ${ }^{\circledR}$, Mundo Animal, São Paulo, Brasil) $(30 \mathrm{mg} / \mathrm{kg} / \mathrm{BID} / 10$ dias), meloxicam (Maxicam - Ourofino Cravinhos São Paulo,Brasil) $(0,1 \mathrm{mg} / \mathrm{kg} / \mathrm{SID} / 5$ dias $)$, dipirona

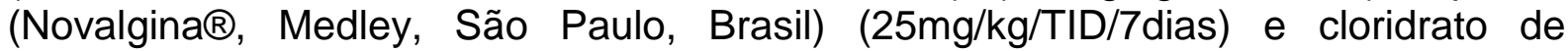
tramadol (Tramadol®, Teuto, Goiás, Brasil) (4mg/kg/TID $/ 5$ dias).

O animal recebeu alta logo após o procedimento cirúrgico com restrição de atividade física e imobilização de conforto por 20 dias. Os pontos foram removidos aos 10 dias. O animal foi avaliado quanto ao grau de claudicação aos 10, 20, 30 e 60 dias do pós-operatório. As avaliações clínicas foram realizadas a cada retorno, na qual foram observados a postura do animal, a deambulação e a sensibilidade dolorosa do membro acometido mediante palpação.

As avaliações radiográficas foram realizadas no pós-operatório imediato e a cada 30 dias até a obtenção da união óssea. Foi avaliado o ângulo obtido no pósoperatório, o avanço da tuberosidade da tíbia após a rotação, a perpendicularidade entre a linha do platô tibial e a linha do ligamento patelar, além da união óssea da osteotomia. 


\section{RESULTADOS}

Durante a avaliação clínica na primeira troca de tala, passados 10 dias do procedimento cirúrgico, foi observado dor articular e o animal apresentava-se poupando o membro, com apoio intermitente. Com 20 dias de pós-operatório o animal retornou para retirada da tala e apresentava-se com claudicação e apoio firme, o movimento de gaveta ainda permanecia presente, porém sem dor e teste de compressão tibial negativo. Aos 60 dias do procedimento pôde ser observado apoio firme livre de claudicação.

Os exames radiográficos foram realizados no pós-operatório imediato e aos 30 e 60 dias após o procedimento. No exame radiográfico realizado logo após a cirurgia foi consignado a mensuração do ângulo do platô tibial final e encontrado $13,6^{\circ}$ e ângulo do platô tibial com o tendão patelar de $90^{\circ}$. Nos 30 dias de pósoperatório, ao exame radiográfico foram observadas uma fratura distal na tuberosidade da tíbia e outra na porção proximal da fíbula. Contudo, não foi observada nenhuma alteração nas mensurações dos ângulos, o que denota que as fraturas supracitadas não causaram nenhuma alteração significativa. $\mathrm{Na}$ imagem radiográfica médio-lateral, 60 dias pós procedimento cirúrgico foi possível a visibilização da união óssea (Figura 3).

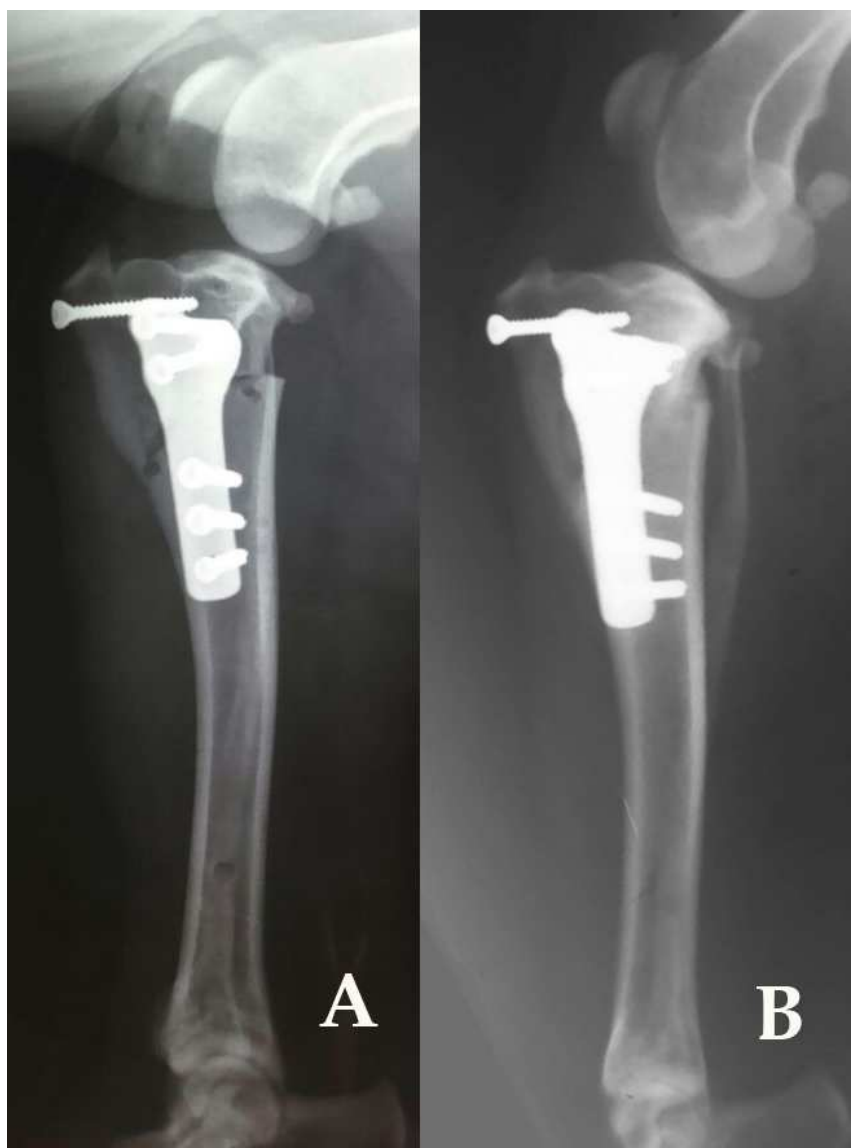

FIGURA 3. Imagens radiográficas em projeção mediolateral de procedimento pós-cirúrgico do ângulo de correção baseado no CORA. A: pós-cirúrgico imediato e B: local para osteotomia da tuberosidade da tíbia e B: 60 dias pós procedimento cirúrgico foi possível a visibilização da união óssea. 


\section{DISCUSSÃO}

Existe uma miríade de técnicas para o tratamento da DLCC (COMERFORD et al., 2013). Este estudo foi balizado nas técnicas, da TPLO, da CBLO e da TTO e todas elas têm como objetivo reduzir a força de compressão tibial pelo nivelamento do platô da tíbia. A CBLO é uma técnica que se fundamenta na mensuração do CORA, e tem como vantagem adicional manter o platô tibial angulado com o tendão patelar em aproximadamente $90^{\circ}$ que gera maior estab ilidade ao joelho (RASKE et al., 2013). Segundo RASKE et al., (2013) o ângulo de rotação do platô tibial recomendado deve ser entre $12-14^{\circ}$, pois o ângulo de $5^{\circ}$ que é obtido na cirurgia de TPLO acaba por causar stress intra-articular a longo prazo. DAMUR et al., (2003) em seu estudo encontrou um ângulo entre $10-15^{\circ}$ sendo s uficiente para manter o platô tibial perpendicular ao tendão patelar e assim neutralizar a compressão tibial. $O$ mesmo sendo alcançado neste caso ora reportado, onde o teste de compressão tibial também foi negativo. No entanto, a importância do TPA no resultado funcional foi questionamento de CHISTOPHER et al., (2014) em estudo com animais tratados com TPLO, relacionando a precisão da osteotomia através de instrumental especifico e a alteração de TPA no pós-operatório. Contudo, RASKE et al., (2013), relataram que a CBLO provou ser um método eficaz para evitar a mudança de TPA no pós-operatório. A angulação de cunha encontrada por MOLES et al., (2009) em estudo, foi de 13,6 graus o que diferiu do encontrado por BRUCE et al., (2007) que relataram 11,5 graus de média do ângulo da cunha. Segundo MOLES et al., (2009) essa variação se deu devido a imprecisão no posicionamento da radiografia e também pela mudança na fórmula utilizada para obtenção do ângulo da cunha.

O planejamento da posição e o raio para realização da osteotomia se dá a partir de projeção radiográfica médio-lateral e para que não se observem alterações nos resultados as medições dos ângulos do platô tibial devem ser mensuradas por apenas um único observador (RASKE et al., 2013, CHISTOPHER et al., 2014). Ratifica-se, portanto, que toda a idealização deste procedimento em questão se deu através destas mesmas projeções radiográficas e com apenas um observador para confecção da medida de rotação, com intuito de evitar alterações. No presente relato o exame radiográfico com projeções médio lateral também foram utilizadas para acompanhar da cicatrização óssea. Segundo RASKE et al., (2013), a não realização de radiografias em projeções crânio-caudais para avaliar a forma da osteotomia da tíbia e a rotação do segmento pode interferir na interpretação da cicatrização óssea. A osteotomia da TT para o avanço foi determinado da mesma forma de BRUCE et al., (2007) ou seja, 1/3 do avanço da TT foi provocado por 2/3 da rotação necessária para a CBLO conforme empregada por RASKE et al., (2013).

Avaliações radiográficas foram realizadas nos seguintes momentos: imediatamente após o procedimento cirúrgico e aos 30 e 60 dias aproximadamente de pós-operatório. $\mathrm{Na}$ projeção radiográfica de 30 dias após cirurgia foram visibilizados fraturas na região distal da crista da tíbia e região próxima da fíbula. $\mathrm{Na}$ literatura esses tipos de fraturas associados a pontos de inserção do pino para auxiliar na rotação e do "JIG" na TPLO e no orifício para o guia de osteotomia da crista da tíbia são possíveis de ocorrer (BRUCE et al., 2007, TAYLOR et al., 2011, FLYNN et al., 2014). No presente relato, aos 60 dias após o procedimento cirúrgico pôde ser visibilizado pelo exame radiográfico total cicatrização óssea. Vários estudos avaliaram o tempo de cicatrização da osteotomia como RASKE et al., 
(2013) que relataram que a cicatrização óssea pós osteotomia é superior ao tempo da TPLO. Em contraponto, CONKLING et al., (2010) em estudo relataram melhor união óssea, oito semanas através da imagem radiográfica. Entretanto, em outro estudo, os autores reportaram que a equivalência do apoio entre os membros, afetados e os normais, só foi atingida por volta das 12 semanas do procedimento cirúrgico (DE MEDEIROS et al., 2011).

Algumas complicações foram relatadas em determinadas técnicas; quais sejam: na CBLO, RASKE et al., (2013) descreveram duas complicações relacionadas a quebra e migração do parafuso e alterações como: infecção secundaria, seroma, afrouxamento de implantes e luxação de patela que foram relatados por outros estudos, mas não foram observados no paciente deste relato (ARTHURS \& LANGLEY-HOBBS, 2007, BRUCE et al., 2007, CHRISTOPHER et al., 2011). Segundo CHRISTOPHER et al., (2011), a patologia meniscal no pósoperatório é uma das principais complicações com taxa de ocorrência de aproximadamente $12 \%$. Portanto, atualmente recomenda-se a retirada do menisco de cães com DLCC que serão tratados com TPLO (RITZO et al., 2014). BERGH \& PEIRONE (2012) relataram que a identificação de fatores de riscos conhecidos ou suspeitos e falha na técnica trans-operatória cursam com a necessidade de ações imediatas de forma a minimizarem-se as complicações no pós-operatório.

\section{CONCLUSÃO}

A técnica resultante da combinação de CBLO e TTO demonstrou ser tão eficaz comparativamente às técnicas anteriores no que tange ao tratamento da deficiência do ligamento cruzado cranial por proporcionar neutralização do impulso cranial da tíbia e manutenção do ângulo do platô tibial.

\section{REFERENCIAS}

ARTHUS, G. I.; LANGLEY-HOBBS, S. J. Patellar luxation as a complication of surgical intervention for the management of cranial cruciate ligament rupture in dogs. Veterinary and Comparative Orthopaedics and Traumatology. v. 20, p. $204-$ 210, 2007.

BERGH, M. S.; PEIRONE, B. Complications of tibial plateau levelling osteotomy in dogs. Veterinary and Comparative Orthopaedics and Traumatology. v. 25, p. 349-358, 2012.

BRUCE, W. J.; ROSE, A.; TUKE, J.; ROBINS, G. M. Evaluation of the triple tibial osteotomy. A new technique for the management of the canine criciate-deficient stifle. Veterinary and Comparative Orthopaedics and Traumatology. v. 20, 2007.

CHRISTOPHER, S. A.; BEETEM, J.; COOK, J. J. Comparison of long-term outcomes associated with three surgical techniques for treatment of cranial cruciate ligament disease in dogs. Veterinary Surgery. v. 42, p. $329-334,2013$. 
COMERFORD, E.; FORSTER,K.; GORTON,K.; MADDOX, T. Management of cranial cruciate ligament rupture in small dogs: A questionnaire study. Veterinary and Comparative Orthopaedics and Traumatology. v. 26, p. 493-497, 2013.

CONKLING, A. L.; FAGIN, B.; DAYER, R. M. Comparison of tibial plateau angle chanGes after tibial plateau leveling osteotomy fixation with conventional or locking screw technology. Veterinary Surgery. v.39, p. $475-481,2010$.

COOK, J. L. Cranial cruciate ligament disease in dogs: biology versus biomechanics. Veterinary Surgery. v. 39, p. $270-277,2010$.

DAMUR, D. M.; TEPIC, S.; MONTAVON, P. M. Proximal tibial osteotomy for the repair of cranial cruciate-deficient stifle joints in dogs. Veterinary and Comparative Orthopaedics and Traumatology. V. 16, p. 211-216, 2003.

DE MEDEIROS, M.; BUSTINDUY, M.S.; RADKE, H.; LANGLEY-HOBBS, S.; JEFFERY. N. Early kinematic outcome after treatment of cranial cruciate ligament rupture by tibial plateau levelling osteotomy in the dog. Veterinary and Comparative Orthopaedics and Traumatology. v. 24, p. 178-184, 2011.

FERRIGNO, C. R. A.; CUNHA, O.; MARIANI, T. C.; IZQUIERDO, D.; DELLA NINA, M. I.; ITO, K. C.; FERRAZ, V. C. M.; ROMANO, L. Tibial tuberosity advancement (TTA): o que é esta nova técnica para tratamento de ruptura de ligamento cruzado cranial em cães. Revista da Anclivepa São Paulo. p. 21 - 23, 2009.

FLYNN, P.; DUNCAN, C. G.; PALMER, R. H.; DUERR, F.M. In Vitro Incidence of fibular penetration with nad without the use of a JIG during tibial plateau leveling osteotomy. Veterinary Surgery. v. 43, p. $495-499,2014$.

GRIFFON, D. J. A Review of the pathogenesis of canine cranial cruciate ligament disease as a basis for future preventive strategies. Veterinary Surgery. v. 39, p. 399-409, 2010.

KIM, S. E.; POZZI, A.; KOWALESKI, M. P.; LEWIS, D. Tibial osteotomies for cranial cruciate ligament insufficienncy in Dogs. Veterinary Surgery. v. 37, p. $111-125$, 2008.

MILOVANCEV, M.; SCHAEFER, S. L. Tibial plateau leveling osteotomy In: MUIR, P. Advances In The Canine Cranial Cruciate Ligament. 1ed. Ames: Wiley-Blackwell. 2010. 289p, cap. 25, p 169-174.

MOLES, A. D.; HILL, T. P.; GLYDE, M. Triple tibial osteotomy for treatment of the canine cranial criciate ligament-deficient stifle joint. Veterinary and Comparative Orthopaedics and Traumatology. V. 22, p. 473-478, 2009.

PIERMATTEI, D. L.; FLO, G. L.; DECAMP, C. E. Manual de ortopedia e tratamento de fraturas de pequenos animais. 4ed. São Paulo: Manole, 2006. 
RASKE, M.; HULSE, D.; BEALE, B.; SAUNDERS, W. B.; KISHI, E.; KUNZE, C. Stabilization of the CORA based leveling osteotomy for treatment of cranial cruciate ligament injury using a bone plate augmented with a headless compression screw. Veterinary Surgery. v.42, p.759-764, 2013.

REIF, U.; DEJARDIN, L. M.; PROBST, C. W.; DECAMP, C. E.; FLO, G. L.; JOHNSON, A. L. Influence of positioning and measurement method on the magnitude of the tibial plateau angle. Veterinary Surgery. v.33, p. $368-375,2004$.

REIF,U.; PROBST, C.W. Comparison of tibial plateau angles in normal and cranial cruciate deficient stifles of labrador retrievers. Veterinary Surgery. v.32, p. $385-$ 389,2003

RITZO, M. E.; RITZO, B. A.; SIDDENS, A. D.; SUMMERLOTT, S.; COOK, J. L. Incidence and type of meniscal injury and associated long-term clinical outcomes in dogs treated surgically for cranial cruciate ligament disease. Veterinary Surgery. v.9999, p. 1-7, 2014.

SCHULZ, K. Diseases oh the joints. In: FOSSUM, T. W. Small animal surgery. 3 ed. Missouri: Mosby Elsevier, 2007, p.1143-1315.

TAYLOR, J.; LANGENBACH, A.; MARCELLIN-LITTLE, D. J. Risk factors for fibular fracture after TPLO. Veterinary Surgery. v. 40, p. $687-693,2011$.

VASSEUR, P. B. Articulação do joelho. In: SLATER, D. Manual de cirurgia de pequenos animais. V. 2. 3ed. São Paulo: Manole, 2007. Cap. 147, p. 2090- 2132. 